\title{
Avaliação morfológica de Carapichea ipecacuanha (Brot.) L. Andersson
}

\author{
Morphological evaluation of Carapichea ipecacuanha (Brot.) L. Andersson \\ Evaluación morfológica de Carapichea ipecacuanha (Brot.) L. Andersson
}

Recebido: 20/04/2021 | Revisado: 29/04/2021 | Aceito: 12/05/2021 | Publicado: 28/05/2021

\author{
Meiciane Ferreira Campelo \\ ORCID: https://orcid.org/0000-0001-7511-4377 \\ Universidade Federal do Pará, Brasil \\ E-mail: meicianecampelo@gmail.com \\ Osmar Alves Lameira \\ ORCID: https://orcid.org/0000-0001-8370-8562 \\ Embrapa Amazônia Oriental, Brasil \\ E-mail: osmar.lameira@embrapa.br \\ Ruanny Karen Vidal Pantoja Portal Moreira \\ ORCID: https://orcid.org/0000-0002-9566-3741 \\ Universidade Federal do Pará, Brasil \\ E-mail: ruanny_vidal@hotmail.com \\ Allan Christiam Santos Ramires \\ ORCID: https://orcid.org/0000-0002-0953-7221 \\ Universidade Federal Rural da Amazônia, Brasil \\ E-mail: allanramires15@gmail.com
}

\begin{abstract}
Resumo
O objetivo desse trabalho foi avaliar e determinar os descritores morfológicos de acessos de ipeca. Foram avaliados 66 acessos de ipeca conservados in vivo na Embrapa Amazônia Oriental, em Belém, PA, totalizando 330 indivíduos, para cinco caracteres morfológicos quantitativos. Os dados foram submetidos as analises multivariadas, realizado a seleção direta e seleção com reanálise agrupadas em dois métodos e com base na distância Euclidiana média utilizada na formação dos agrupamentos pelos métodos Unweighted Paired Group Method Using ArithmeticAverages UPGMA de otimização de Tocher e para análise de componentes principais. Dos cinco caracteres avaliados segundo os dois procedimentos utilizados nenhum se mostrou redundante, logo não houve descartes. Com base na distância Euclidiana média apresentaram três menores distâncias 0,09; 0,17 e 0,20 entre pares de acessos e permitiram separálos em três grupos divergentes pelo método UPGMA e pelo método de Tocher houve a formação de dois grupos divergentes. Três caracteres apresentaram as maiores contribuições para a divergência, a largura da folha, comprimento do pecíolo e altura da planta, com 80,04 \%. Diante disso, todos os caracteres apresentaram contribuição para diferenciação entre acessos os acessos de C. Ipecacuanha, sendo largura da folha, comprimento do pecíolo e altura da planta os que respectivamente mais contribuíram para a divergência entre os acessos.
\end{abstract}

Palavras-chave: Ipeca; Banco ativo de germoplasma; Caracterização; Variação morfológica.

\begin{abstract}
The aim of this work was to evaluate and determine the morphological descriptors of accessions of ipecac. 66 accessions of ipecac preserved in vivo at Embrapa Amazônia Oriental, in Belém, PA, were evaluated, totaling 330 individuals, for five quantitative morphological characters. The data were submitted to multivariate analyzes, direct selection and selection with reanalysis were carried out grouped in two methods and based on the average Euclidean distance used in the formation of the clusters by the methods Unweighted Paired Group Method Using ArithmeticAverages -UPGMA for Tocher's optimization and for analysis of main components. Of the five characters evaluated according to the two procedures used, none was redundant, so there were no discards. Based on the average Euclidean distance, they presented three shortest 0.09 distances; 0.17 and 0.20 between pairs of accessions and allowed to separate them into three divergent groups by the UPGMA method and by the Tocher method there was the formation of two divergent groups. Three characters presented the greatest contributions to the divergence, the leaf width, petiole length and plant height, with $80.04 \%$. In view of this, all characters contributed to differentiate between accessions and accessions of C. Ipecacuanha, with leaf width, petiole length and plant height being the ones that most contributed to the divergence between accessions.
\end{abstract}

Keywords: Ipeca; Active germplasm bank; Description; Morphological variation.

\section{Resumen}

El objetivo de este trabajo fue evaluar y determinar los descriptores morfológicos de accesiones de ipecacuana. Se evaluaron 66 accesiones de ipeca preservadas in vivo en Embrapa Amazônia Oriental, en Belém, PA, totalizando 330 individuos, se estudiaron cinco caracteres morfológicos cuantitativos. Los datos se sometieron a análisis multivariados, la selección directa y la selección con reanálisis se llevaron a cabo agrupados en dos métodos y con 
base en la distancia euclidiana promedio utilizada en la formación de los conglomerados por los métodos Método de grupo emparejado no ponderado mediante promedios aritméticos -UPGMA para optimización de Tocher para análisis de componentes principales. De los cinco caracteres evaluados según los dos procedimientos utilizados, ninguno demostró ser redundante, por lo que no hubo descartes. Con base en la distancia euclidiana promedio, presentaron tres distancias más cortas de 0.09; 0,17 y 0,20 entre pares de accesiones y se permitió separarlos en tres grupos divergentes por el método UPGMA y por el método de Tocher se produjo la formación de dos grupos divergentes. Tres caracteres presentaron las mayores contribuciones a la divergencia, ancho de hoja, largo del pecíolo y altura de planta, con $80.04 \%$. En vista de esto, todos los caracteres contribuyeron a diferenciar entre accesiones y accesiones de C. Ipecacuanha, siendo el ancho de la hoja, el largo del pecíolo y la altura de la planta los que más contribuyeron a la divergencia entre accesiones.

Palabras clave: Ipeca; Banco de germoplasma activo; Descripción; Variación morfológica.

\section{Introdução}

A Carapichea ipecacuanha (Brot) L.Andersson é conhecida popularmente como ipeca, sendo uma referência entre as espécies de plantas medicinais com princípios ativos de relevância econômica em risco de extinção, devido à excessiva extração comercial (Junior et al.,2012).A ipeca encontra-se na lista de espécies ameaçadas de erosão genética ou em vias de extinção, por ter sofrido intenso processo extrativo nos últimos dois séculos, pela abertura de novas fronteiras agrícolas e outros fatores, tendo suas áreas de ocorrência natural reduzida (Lameira, 2002), uma vez que para a obtenção da emetina e cefalina, demandadas pela indústria farmacêutica, faz-se a retirada da raiz da planta, pois é a parte que possui maior concentração desses alcalóides (Rocha \& Lameira, 2011).

O potencial econômico de espécies medicinais nativas no Brasil é imenso, tanto que tais espécies são consideradas uma riqueza a ser preservada e utilizada (Pereira et al., 2006). Para aprimorar o uso desses recursos medicinais nativos, são indispensáveis que as mesmas sejam incluídas em um programa de conservação. Os Bancos Ativos de Germoplasma (BAGs) são uma alternativa para a conservação dos recursos genéticos vegetais (Costa et al., 2011).

Em virtude disso, a Instituição da Embrapa Amazônia Oriental localizada no município de Belém-PA, no ano de 1988 instalou o Banco Ativo de Germoplasma (BAG) de Ipeca com o objetivo de introduzir, coletar, conservar, caracterizar, documentar e disponibilizar o germoplasma dessa espécie (Pádua et. al., 2020) nesse banco os acessos coletados de diferentes procedências nos permite conduzir estudos na área de caracterização e avaliação morfológica.

Uma boa avaliação de materiais de um Bag e a determinação da distância genética entre os acessos é de grande relevância na identificação e seleção de genótipos de interesse para os programas de melhoramento genético (Sudré et al., 2010).Na avaliação de acessos em bancos de germoplasma a observação e avaliação de um grande número de características em um mesmo individuo são muito comuns, principalmente quando a quantidade de indivíduos é grande, e devido a isso se lança mão de técnicas multivariadas para análise dessas características. As técnicas multivariadas são métodos que avaliam simultaneamente as múltiplas características de cada indivíduo, e as classificam de forma que a interpretação seja mais rápida. Dentre estas estão à análise de agrupamento, análise de componentes principais (Mingoti, 2007).

Portanto, o objetivo desse trabalho foi determinar descritores morfológicos de acessos de ipeca visando à avaliação dos germoplasmas.

\section{Metodologia}

Refere-se a um estudo realizado em campo e de cunho quantitativo segundo as descrições de Pereira et al., (2018). Este estudo foi realizado no Banco Ativo de Germoplasma (Bag) de Ipeca, instalado no Horto de Plantas Medicinais da Embrapa Amazônia Oriental, com altitude de $10 \mathrm{~m}$ e temperatura média anual de $30^{\circ} \mathrm{C}$. O clima em Belém, segundo a série histórica da cidade de Belém, apresenta precipitação anual média de $3.070 \mathrm{~mm}$, entretanto, as chuvas se distribuem de maneira 
diferente durante o ano, formando um período muito chuvoso (dezembro a maio) e um período menos chuvoso (junho a novembro) Lira et al., (2020).

Foram avaliados 66 acessos de Carapichea ipecacuanha (Brot) L. Andersson, conservados in vivo, cultivados em canteiros sob telado de sombrite $50 \%$ de luminosidade, os tratos culturais e fitossanitários foram realizados de acordo com a necessidade da espécie. Foram selecionadas cinco plantas por acesso de forma aleatória e marcados com fita. Os acessos possuem origem de seis Estados do Brasil, sendo um acesso da Bahia- BA, dois do Espírito Santo- ES, um de Minas GeraisMG, vinte e nove de Mato Grosso - MT, três do Rio de Janeiro-RJ, vinte e trinta de Rondônia - RO (Tabela 1).

Tabela 1. Procedência e nomenclatura de 66 acessos conservados no Bag de ipeca da Embrapa Amazônia Oriental.

\begin{tabular}{cl}
\hline Procedência & Nomenclatura (Cod. Local) \\
\hline Bahia & 767 \\
Espirito Santo & $759 ; 761$ \\
Minas Gerais & 753 \\
& \\
Rio de Janeiro & $745 ; 746 ; 751$ \\
& \\
& $571 ; 572 ; 573 ; 574 ; 575 ; 576 ; 577 ; 580 ;$ \\
& $589 ; 590 ; 594 ; 595 ; 596 ; 602 ; 607 ; 689 ;$ \\
& $690 ; 693 ; 694 ; 695 ; 696 ; 700 ; 701 ; 702 ;$ \\
Mato Grosso & $707 ; 708 ; 712 ; 714 ; 719$ \\
& $610 ; 612 ; 775 ; 776 ; 777 ; 779 ; 801 ; 802 ;$ \\
& $803 ; 806 ; 807 ; 808 ; 810 ; 811 ; 812 ; 814 ;$ \\
& $815 ; 816 ; 819 ; 826 ; 827 ; 828 ; 829 ; 833 ;$ \\
\hline
\end{tabular}

Fonte: ALELO (2021).

Para a seleção de descritores e divergência genética entre os acessos os caracteres morfológicos quantitativos avaliados foram: altura da planta; altura da primeira ramificação; largura da folha; comprimento da folha; e comprimento do pecíolo. As medições foram realizadas com auxílio de régua e paquímetro.

Após tabulação dos dados, foi realizada análise segundo dois métodos com o auxílio do software $\mathrm{R}(2020)$ para identificação de possíveis caracteres quantitativos redundantes ou invariáveis para posterior seleção dos descritores, o primeiro método foi o de seleção direta, proposto por Jolliffe (1937) neste caso eliminam-se todos os caracteres que apresentarem maior coeficiente de ponderação em valor absoluto (autovetor) no componente principal de menor autovalor, partindo do último componente até aquele cujo autovalor não excedesse a 0,70. O segundo método foi à seleção com reanálise, sugerida por Cury (1993) em que a cada caráter sugerido para descarte, realizou-se nova análise com os caracteres remanescentes, examinando os coeficientes de correlação do caráter sugerido para descarte com os demais caracteres, sendo finalizada quando o caráter a ser descartado se mostrou altamente correlacionado com pelo menos um caráter já descartado. O descarte final será realizado com base na informação obtida simultaneamente nos dois procedimentos.

Os dados dos descritores selecionados foram empregados para a obtenção das dissimilaridades entre cada par de acessos com base na distância euclidiana média padronizada, no mesmo software, uma vez que os acessos encontram-se estabelecidos sem obedecer a nenhum delineamento experimental. 
A matriz de distâncias obtidas foi utilizada na formação dos agrupamentos pelos métodos de UPGMA, otimização de Tocher e para análise de componentes principais.

\section{Resultados e Discussão}

Dos cinco caracteres avaliados segundo os dois procedimentos utilizados nenhum apresentou redundância, logo não houve descartes. A eliminação de caracteres redundantes é uma decisão vantajosa, o descarte quando realizado deverá proporcionar, redução no tempo, na mão de obra e nos custos das atividades de avaliação e caracterização em bancos e coleções de germoplasma dessa espécie (Lameira et al., 2020).Logo, neste trabalho foram realizados os procedimentos de seleção direta, proposto por Jolliffe (1937) e a seleção com reanálise, sugerida por Cury (1993), entretanto os testes não identificaram redundância que justificassem possíveis descartes.

A matriz de dissimilaridade obtida entre os pares de 66 acessos de C. ipecacuanha gerada com base em cinco caracteres quantitativos revela maior semelhança, ou seja, maior similaridade e menor distância $(0,09)$ entre os acessos $775 \times$ 777 , segunda menor distância $(0,17)$ entre os pares 701 x 576, e terceira menor distância $(0,20)$ entre os pares 602 x 589, a alta similaridade encontradas entre esses pares de acesso confere a eles baixa variabilidade.

Com base no método UPGMA os 66 acessos formaram três grupos divergentes: o grupo I contendo os acessos 776 coletados em RO, o grupo II composto pelos acessos 833-RO, 707-MT, 806-RO, 829-RO, e o grupo III composto pelos demais acessos, com diferentes procedências (Figura 1). Vale ressaltar que a relação da procedência geralmente não resulta como indicador de diversidade genética, pois em muitos casos a separação geográfica geralmente não resulta em maior distância genética (Cruz \& Carneiro, 2003; Arriel et, al., 2006), assim como o que foi constatado, neste trabalho, onde o grupo II possui quatro acesso de dois Estados e o grupo III constituído por sessenta e um acessos com procedência em seis Estados. 
Figura 1.Dendograma gerado pelo método UPGMA a partir das dissimilaridades obtidas entre 66 acessos de ipeca conservados no bag da Embrapa Amazônia Oriental, com base em cinco caracteres morfológicos.

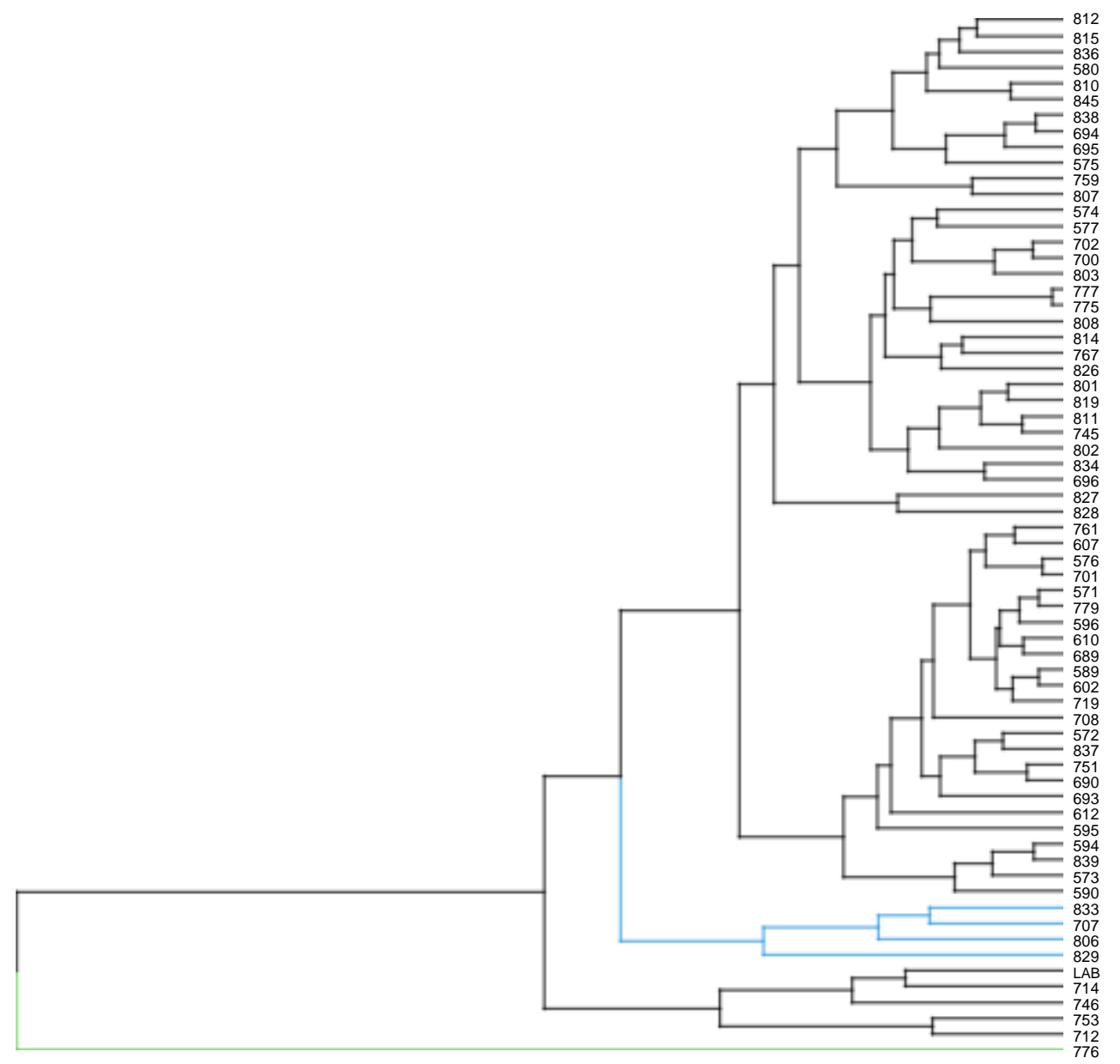

Fonte: Autores.

Pelo método de Tocher houve a formação de dois grupos divergentes entre os 66 acessos (Tabela 2). No grupo I está presente apenas o acesso 776 cuja procedência é Rondônia; o grupo II agrupa os demais acessos com procedências em diferentes localidades. Observou-se alguma semelhança na formação dos grupos com a análise pelo método do UPGMA, ressalta-se a formação de um grupo contendo um único acesso (776). A formação de distintos grupos evidência a variabilidade genética existente na população, uma vez que a formação de agrupamentos de acessos demonstra uma similaridade alta entre os mesmo (Martins, 2011). 
Tabela 2. Grupos formados pelo método de Tocher entre os 66 acessos de ipeca conservados no bag da Embrapa Amazônia Oriental, com base em cinco caracteres morfológicos.

\begin{tabular}{|c|c|c|c|c|c|c|c|}
\hline \multirow{2}{*}{$\begin{array}{l}\text { Grupos } \\
\text { I }\end{array}$} & \multicolumn{7}{|c|}{ Acessos } \\
\hline & 776 & & & & & & \\
\hline \multirow{10}{*}{ II } & 775 & 777 & 811 & 745 & 819 & 702 & 700 \\
\hline & 801 & 803 & 826 & 814 & 834 & 696 & 802 \\
\hline & 759 & 767 & 577 & 574 & 808 & 812 & 807 \\
\hline & 695 & 580 & 815 & 838 & 694 & 810 & 837 \\
\hline & 572 & 845 & 575 & 573 & 836 & 607 & 690 \\
\hline & 576 & 701 & 761 & 779 & 751 & 596 & 571 \\
\hline & 602 & 689 & 708 & 589 & 839 & 610 & 693 \\
\hline & 594 & 719 & 612 & 590 & 595 & 753 & 828 \\
\hline & 827 & 806 & 712 & 707 & 833 & LAB & 829 \\
\hline & 714 & 746 & & & & & \\
\hline
\end{tabular}

Fonte: Autores.

A análise de componentes principais vem se destacando como uma metodologia importante na contribuição de variação total disponível entre os indivíduos analisados fornece indicação para eliminar componentes que pouco contribui sendo responsáveis por direções que estão associadas à baixa variabilidade (Pereira,1989, Hongyu et al., 2016).

A largura da folha, comprimento do pecíolo e altura da planta foram os caracteres que mais contribuíram para a divergência entre os 66 acessos com percentuais de contribuição igual a 29,42\%; 26,94\%; 23,68\% respectivamente (Tabela 3). Assis \& Giulieti (1999) em um estudo comparativo de populações de C. ipecacuanha, afirmam que as variações associadas ao comprimento e largura das folhas, estão expressas na variação das folhas, na qual variaram desde elípticas até ovais, oblongolanceoladas ou obovais. Resultados semelhantes foram observados por Neves et al., (2017) cujo o descritor que mais contribuiu para a divergência entre os acessos de Psychotria ipecacuanha (Brotero) Stokes foi Altura da planta (44,23\%), as procedências do material estudo abordaram três estados brasileiros e com espécimes in vivo.

O componente que menos contribuiu foi o caráter altura da primeira ramificação com $1,24 \%$, logo esta relacionada à baixa divergência. Esse resultado diverge do encontrado por Neves et al (2017) em estudo com 17 acessos de ipeca, no qual o caracter que menos contribuiu para a divergência entre acessos foi comprimento do pecíolo. 
Tabela 3. Contribuição relativa de cinco caracteres para a dissimilaridade de 66 acessos de ipeca conservados no bag da Embrapa Amazônia Oriental, com base em cinco caracteres morfológicos.

\begin{tabular}{ll}
\hline Caráter & Contribuição (\%) \\
\hline Altura da planta & 23,68 \\
Altura $1^{\circ}$ ramificação & 1,24 \\
Largura da folha & 29,42 \\
Comprimento da folha & 18,72 \\
Comprimento do pecíolo & 26,94 \\
\hline
\end{tabular}

Fonte: Autores.

Segundo Lameira et al., (2020) os descritores quantitativos são importantes na avaliação de germoplasma de ipeca pois, podem disponibilizar informações primordiais para o melhoramento genético, entretanto, resultados devem ser complementados com estudos posteriores de caracteres qualitativos.

\section{Conclusão}

Este estudo avaliou a variabilidade dos caracteres quantitativos: altura da planta; altura da primeira ramificação; largura da folha; comprimento da folha e comprimento do pecíolo, todos apresentaram contribuição para diferenciação entre acessos de C. ipecacuanha.

Identificou e agruparam os descritores, os que mais contribuíram para a divergência entre os acessos foram largura da folha, comprimento do pecíolo e altura da planta, mostrando-se importantes na avaliação de germoplasma de ipeca. Recomenda-se a soma de estudos de caracteres morfológicos qualitativos para a espécie.

Estas informações são cruciais para a espécie auxiliar no conhecimento e uso da variabilidade genética e servirão de base para a seleção de indivíduos de interesse em programas de melhoramento, além de fornecer informações fundamentais que facilitará a identificação de espécimes em ecossistemas nativos ou plantados.

\section{Agradecimentos}

Os autores agradecem à Coordenação de Aperfeiçoamento de Pessoal de Nível Superior (CAPES) pelo apoio e bolsa de estudo de Doutorado, à Embrapa Amazônia Oriental por ceder o local e a estrutura, e ao Programa de Doutorado da Rede Bionorte- Biodiversidade e Biotecnologia.

\section{Referências}

Arriel, N. H. C., DI Mauro, A. O., DI Mauro, S. M. Z., Bakke, O. A., Unêda-Trevisoli, S. H., Costa, M. M., \& Corrado, A. R. (2006). Técnicas Multivariadas na Determinação da diversidade genética em gergelim usando marcadores RAPD. Pesquisa agropecuária brasileira, 41(5), 801-809.

Assis, M. C. D., \& Giulietti, A. (1999). Diferenciação morfológica e anatômica em populações de" ipecacuanha"-Psychotria ipecacuanha (Brot.) Stokes (Rubiaceae). Brazilian Journal of Botany, 22(2), 205-216.

Costa, T. S., Silva, A. V. C. D., Lédo, A. D. S., Santos, A. R. F. D., \& Silva Júnior, J. F. D. (2011). Diversidade genética de acessos do banco de germoplasma de mangaba em Sergipe. Pesquisa Agropecuária Brasileira, 46(5), 499-507.

Cruz, C. D., Regazzi, A.J., \& Carneiro, P. C. S. (2004). Modelos Biométricos Aplicados ao Melhoramento Genético. editora UFV.

Cruz, C. D., \& Souza Carneiro, P. C. (2006). Modelos biométricos aplicados ao melhoramiento genético (No. 575.1015195). Universidad Federal de Viçosa.

Cury, R. (1993). Dinâmica evolutiva e caracterizaçao de germoplasma de mandioca (Manihot esculenta, Crantz) na agricultura autóctone do Sul do Estado de Sao Paulo (Doctoral dissertation, Universidade de São Paulo). 
Daher, R. (1993). Diversidade morfológica e isoenzimática em capim elefante (Pennisetum purpureum Schum.). 1993.110 f(Doctoral dissertation, Dissertação (Mestrado em Genética e Melhoramento de Plantas) Universidade Federal de Viçosa, Viçosa, MG).

Embrapa. (2021). Embrapa Recursos Genéticos E Biotecnologia. Núcleo de Tecnologia da Informação (NTI). AleloVegetal.

Hongyu, K., Sandanielo, V. L. M., \& de Oliveira Junior, G. J. (2016). Análise de componentes principais: resumo teórico, aplicação e interpretação. E\&S Engineering and science, 5(1), 83-90.

Jolliffe, I. T. (1973). Discarding variables in a principal componentanalysis. II: Real data. Journal of the Royal Statistical Society: Series C (Applied Statistics), 22(1), 21-31.

Júnior, W. S. F., Cruz, M. P., dos Santos, L. L., \& Medeiros, M. F. T. (2012). Use and importance of quina (Cinchona spp.) and ipeca (Carapichea ipecacuanha (Brot.) L. Andersson): Plants for medicinal use from the 16th century to the present. Journal of Herbal Medicine, 2(4), $103-112$.

Lameira, O. A. (2002). Cultivo da ipecacuanha [Psychotria ipecacuanha (Brot.) Stokes]. Embrapa Amazônia Oriental-Circular Técnica (INFOTECA-E).

Lameira, O. A., Cordeiro, I. M. C. C., \& Pires, H. C. G. (2020). Avaliação dos Descritores Morfoagronômico e Morfoanatomia da Lâmina Foliar de Pilocarpus: Microphyllus Stapf ex Wardleworth-Rutaceae, Ananas Comosus Var. Erectifolius (LB Smith) Coppens \& F. Leal-Bromeliacea e Psychotria Ipecacuanha (Brot.) Stokes. Editora Appris.

Lira, B. R. P., Lopes, L. D. N. A., das Chaves, J. R., Santana, L. R., \& Fernandes, L. L. (2020). Identificação de Homogeneidade, Tendência e Magnitude da Precipitação em Belém (Pará) entre 1968 e 2018. Anuário do Instituto de Geociências, 43(4), 426-439.

Lobato P. N. R., Lameira. O. A., Oliveira, M. do S. P., de Souza, B., Ilkiu, F., Monfort. F., Elizabeth. L., Correia. R. G., (2017). Caracterización Y Evaluación Morfológica de partes aéreas de accesiones e Psychotria Ipecacuanha (Brot.) Stokes (Ipeca). Revista Cubana De Plantas Medicinales, 22(1), 1-12.

Martins, F. A. (2011). Integration of morphoagronomical, molecular and phytopathological data forsetting of corecollection. 119 F. TESE - Universidade Federal de Viçosa, VIÇOSA, 2011.

Mingoti, S. A. (2007). Análise de dados através de métodos estatística multivariada: uma abordagem aplicada. In Análise de dados através de métodos estatística multivariada: uma abordagem aplicada (pp. 295-295).

Pádua, J. G., Albuquerque, M., \& de Mello, S. C. M. (2020). Bancos e coleções de germoplasma da Embrapa: Conservação e uso. Embrapa Recursos Genéticos e Biotecnologia-Documentos (INFOTECA-E).

Pereira V.A.(1999). Utilização de análise multivariada na caracterização de germoplasma de mandioca ( ManihotesculentaCrantz.) [tese]. Piracicaba: Escola Superior de Agricultura Luiz de Queiroz, Universidade de São Paulo.

Pereira, A. S., Shitsuka, D. M., Parreira, F. J. \&Shitsuka, R. (2018). Metodologia da pesquisa científica. UFSM. 119p. https://repositorio.ufsm.br/bitstream/handle/1/15824/Lic_Computacao_Metodologia-Pesquisa-Cientifica.pdf?sequence=1 .

Pereira, L. P. D., Luz, L. P. D., Tedesco, S. B., \& Silva, A. C. F. D. (2006). Número de cromossomos em populações de Achyrocline satureioides Lam. (marcela) do Estado do Rio Grande do Sul, Brasil. Ciência Rural, 36(2), 678-681.

R Core Team (2020). R: A languageandenvironment for statisticalcomputing. R Foundation for StatisticalComputing, https://www.R-project.org/.

Rocha, T. T., \& Lameira, O. A. (2011). Avaliação do período de floração e frutificação do BAG Ipecacuanha. In Embrapa Amazônia Oriental-Artigo em anais de congresso (ALICE). In: Seminário De Iniciação Científica Da Embrapa Amazônia Oriental, 15., 2011, Belém, PA. A ciência de fazer ciência: anais. Belém, PA: Embrapa Amazônia Oriental, 2011.

Silva, S. G. A., da Silva, F. K. G., Diniz, A. L., \& Arriel, N. (2008). Caracterização da diversidade de acessos de pinhão manso. In Embrapa Algodão-Artigo em anais de congresso (ALICE). In: Congresso Brasileiro De Mamona, 3., 2008, Salvador. Energia e ricinoquímica: anais. Salvador: SEAGRI: Embrapa Algodão.

Sudré, C. P., Gonçalves, L. S. A., Rodrigues, R., Amaral Júnior, A. D., Riva-Souza, E. M., \& Bento, C. D. S. (2010). Genetic variability in domesticated Capsicum spp. as assessed by morphological and agronomic data in mixed statistical analysis. Genetics and molecular research, $9(1), 283-294$. 